\title{
Preparation and cytocompatibility of polylactic acid/hydroxyapatite/ graphene oxide nanocomposite fibrous membrane
}

\author{
MA HaiBin ${ }^{1,3}$, SU WenXin ${ }^{2}$, TAI ZhiXin ${ }^{1}$, SUN DongFei $^{1}$, YAN XingBin ${ }^{1 *}$, LIU Bin $^{2} \&$ \\ XUE QunJi ${ }^{1}$ \\ ${ }^{1}$ State Key Laboratory of Solid Lubrication, Lanzhou Institute of Chemical Physics, Chinese Academy of Sciences, Lanzhou 730000, China; \\ ${ }^{2}$ School of Stomatology, Lanzhou University, Lanzhou 730000, China; \\ ${ }^{3}$ Gansu Provincial Hospital of Traditional Chinese Medicine, Lanzhou 730050, China
}

Received February 23, 2012; accepted May 3, 2012

\begin{abstract}
A series of polylactic acid (PLA) based nanocomposite fibrous membranes, including neat PLA, PLA/hydroxyapatite (HA) and PLA/HA/graphene oxide (GO), were fabricated via electrospinning method. The morphology and composition were investigated by scanning electron microscopy (SEM), X-ray diffraction (XRD) and Fourier transform infrared spectroscopy (FTIR) respectively. The thermal stability was determined by thermogravimetric analysis (TGA). To estimate the cytocompatibility of asprepared PLA/HA/GO fibrous membrane, MC3T3-E1 cells were cultured, and the corresponding cell adhesion and differentiation capability were investigated by fluorescence microscopy, SEM and MTT test. The electrospun ternary PLA/HA/GO membrane exhibited three-dimensional fibrous structure with relatively rough surface morphology, which made itself ideal for cell attachment and proliferation in bone tissue regeneration. The fluorescence microscopy, SEM and MTT test confirmed that the PLA/HA/ GO nanocomposite fibrous membrane created a proper environment for the seeding and proliferation of MC3T3-E1 cells.
\end{abstract}

polylactic acid, hydroxyapatite, graphene oxide, fibrous membranes

Citation: $\quad$ Ma H B, Su W X, Tai Z X, et al. Preparation and cytocompatibility of polylactic acid/hydroxyapatite/graphene oxide nanocomposite fibrous membrane. Chin Sci Bull, 2012, 57: 3051-3058, doi: 10.1007/s11434-012-5336-3

Tissue engineering is a versatile technique that applies the principles of engineering and life sciences to improve tissue biologic function [1], including bone tissue repair and reconstruction [2-4]. This technique involves processes such as seeding cells onto a scaffold, creating artificial organs and tissues, triggering or modulating new tissue formation, etc.

Currently, synthetic biomaterials (bioceramics and biopolymers) have been widely applied to the tissue engineering fields [5-8]. It is known that bone is an organicinorganic compound. Logically, fabrication of hybrid materials that combine strength and stiffness of inorganics with toughness and resorbability of organics is a good way to create considerate materials to tissue engineering [9]. Also, the ideal scaffold must satisfy a number of demands: (1)

*Corresponding author (email: xbyan@licp.cas.cn) appropriate level of porosity allowing for cell migration; (2) appropriate surface chemical and microstructural properties to facilitate cellular attachment, growth, proliferation, and differentiation; (3) proper biodegradation rate that tuned with regeneration rate of the desired natural tissue and without any undesirable by-products $[10,11]$.

It is well known that the electrospinning technique can be used to produce biocompatible polymer fibers with diameters from nanometer to micrometer [12-17]. Recently, the electrospinning technique has been applied to the preparation of the various scaffold materials for the tissue engineering [18]. The structure that mimics the natural extra cellular matrix (ECM) at the nano-, micro- and macroscales, can be beneficial for flexible tissue design and regeneration [19].

Poly lactic acid (PLA), as a synthetic polymer with desirable mechanical property profile $[8,20]$, thermoplastic 
processibility, biocompatibility and biodegradability, is one of the most promising biodegradable polymers for tissue engineering [21,22]. Because of the excellent biocompatibility, bioactivity and osteoconductivity, hydroxyapatite (HA) has been added into PLA matrix to enhance its biocompatibility [23]. Due to the excellent mechanical properties, CNT has been added into PLA to enhance its mechanical strength as well [24,25]. However, CNTs are hardly dispersed into polymer matrix, which limits their application in tissue engineering fields.

Graphene oxide (GO), with extraordinary mechanical properties (high Young's modulus and hardness, and excellent flexibility) and low cost compared to CNT, have been considered to be effective reinforcements for high-performance composites [26-29]. Meanwhile, because of the existence of oxygen-containing groups, GO is easy to be dispersed into some polar solvents and to form intercalated composites with polar molecules through the strong interaction [30-34]. Moreover, GO have been proved to exhibit excellent biocompatibility [35-40].

In this study, PLA/HA/GO nanocomposite fibrous membrane, from a DMF-dichloromethane suspension of PLA, $\mathrm{HA}$ and GO, was fabricated by electrospinning technique. The ultra-fine fibers presented a three-dimensional structure, which was beneficial to cell seeding and cell growth. The PLA/HA/GO fibrous membrane mimiced the natural extra cellular matrix (ECM) structure and the adding of HA and GO promoted the cell's proliferation and differentiation. It makes such nanocomposite fibrous membrane attractive for the applications in tissue engineering field.

\section{Materials and methods}

\subsection{Materials}

PLA $\left(M_{\mathrm{w}}=26000\right)$ was purchased by Shandong Daigang Tech. Co., Ltd., China. Graphite powder (325 meshes) was purchased from Qingdao Huatai Tech. Co., Ltd., China. HA (purity: 99\%; diameter: 20-40 nm) was purchased from Nanjing Nanotech. Co., Ltd., China. Fetal bovine serum (FBS) was purchased from Hangzhou Sijiqing Bio. Engineer. Mater. Co., Ltd., China. MC3T3-E1 cells and RPMI 1640 medium were purchased from Lanzhou Shenggong Biomedical Co., Ltd., China. Trypsin-EDTA solution and 3(4,5-dimethylthiazol-2-yl)-2,5-diphenyltetrazolium bromide (MTT) were purchased from Sigma. Dichloromethane and Dimethylformamide (DMF) were purchased from Tianjin Chemical Reagent Co., Ltd., China. Other reagents were commercially available and were of analytical reagent grade. All chemicals and solvents were used as received.

\subsection{Synthesis of PLA/HA/GO precursor suspension}

GO was prepared according to the method described by Hummers [41] with a modification. In order to prepare
PLA/HA/GO precursor suspension, powdery HA and GO were dispersed in a $5 \mathrm{~mL}$ of DMF and dichloromethane (volume ratio $=2: 3$ ) solution by ultrasonication to form a uniform suspension. After that, PLA was added into the suspension with the aid of stirring. In this precursor suspension, the percentage composition of PLA was $12 \mathrm{wt} \%$ and the weight ratio among PLA, HA and GO was $20: 2: 1$. The ratio of nanoparticles plays a key role in determining the formation of fibrous membrane. In our experiments, we found that the inorganic particles can be dispersed into PLA matrix when the ratio is lower than 9:1 (PLA against $\mathrm{HA}+\mathrm{GO}$ ). Higher content of HA and GO is unfavorable to form the 3D fibrous structure.

In comparison, the same content of PLA was dissolved into another $5 \mathrm{~mL}$ of DMF-dichloromethane solution to form a precursor solution for electrospinning PLA nanofibers, and the same contents of PLA and HA were dispersed into the similar solution to form a precursor dispersion for electrospinning PLA/HA nanofibers.

\subsection{Electrospinning of PLA/HA/GO composite nano- fibers}

In a typical electrospinning process for preparing PLA/HA/ GO nanofibers, the precursor suspension was loaded into a plastic syringe equipped with a 23-gauge stainless steel needle. A high voltage of $15 \mathrm{kV}$ was supplied by a direct-current power supply and the feeding rate for the precursor suspension was adjusted to a constant rate of $3 \mathrm{~mL} / \mathrm{h}$ by using a syringe pump. A piece of aluminum foil was placed $15 \mathrm{~cm}$ below the tip of the needle to collect the asspun nanofibers. Also, neat PLA and PLA/HA nanofibers were prepared under the same electrospinning conditions, respectively.

\subsection{Fabrication of PLA/HA/GO nanocomposite mem- branes}

In order to prepare PLA/HA/GO nanocomposite membranes, the precursor suspension mentioned above were cast on a clean glass plate, and all of the samples were kept in fume hood to remove residual solvent, then cut into pieces of $1 \mathrm{~cm} \times 1 \mathrm{~cm}$ for MTT test.

\subsection{Characterization of as-electrospun nanofibers}

Transmission electron microscopy (TEM, JEOL, JEM-2010) was employed to investigate the morphology of as-prepared GO, using an accelerating voltage of $200 \mathrm{kV}$. XPS measurement of the GO was performed on a Perkin-Elmer PHI-5702 multi-functional X-ray photoelectron spectroscope (Physical Electronics, USA), using Al K $\alpha$ radiation (photon energy $1476.6 \mathrm{eV}$ ) as the excitation source and the binding energy of $\mathrm{Au}\left(\mathrm{Au} 4 \mathrm{f}_{7 / 2}: 84.00 \mathrm{eV}\right)$ as the reference. Field emission scanning electron microscope (FESEM, 
JEOL, JSM 6701F) was employed to investigate the morphology of as-electrospun nanofibers. X-ray diffraction (XRD) patterns were carried out by a X-ray diffraction using $\mathrm{Cu} \mathrm{K} \alpha$ radiation (XRD, Rigaku, D/Max-2400), and Fourier transformation infrared (FTIR) spectra were recorded using a Bruker IFS66V FTIR spectrometer, to investigate the composition of the samples. Raman spectra were recorded using a micro-Raman spectroscopy (JY-HR800, the excitation wavelength of $532 \mathrm{~nm}$ ). Thermal properties of the fibrous samples were measured on a thermogravimetric analysis (TGA-DSA 2960, TA Instruments) under nitrogen atmosphere from 10 to $800^{\circ} \mathrm{C}$ at a heating rate of $10{ }^{\circ} \mathrm{C} / \mathrm{min}$.

\subsection{3T3-E1 cell culture and cell seeding}

MC3T3-E1 cells were maintained at RPMI-1640 with 10\% fetal bovine serum and $1 \%$ antibiotic/antimycotic solution in a $5 \% \mathrm{CO}_{2}$ incubator at $37^{\circ} \mathrm{C}$, refreshed every 3 or $4 \mathrm{~d}$ with Trypsin-EDTA solution, and then re-suspended in a fresh culture medium. The scaffolds were cut into pieces $(1 \mathrm{~cm} \times 1 \mathrm{~cm})$ and soaked in $75 \%$ ethanol for $1 \mathrm{~h}$ to sterilize, after that exchanged with phosphate-buffered saline (PBS) for three times (30 $\mathrm{min}$ each). The scaffolds were then washed with RPMI-1640 containing 10\% FBS for two times ( $2 \mathrm{~h}$ each). The $2 \times 105$ cell suspension was seeded on each scaffold. The cell-scaffold constructs were cultured in RPMI-1640 supplemented with 10\% FBS, 1\% antibiotic/ antimycotic solution for up to $48 \mathrm{~h}$.

\subsection{Cytocompatibility test}

MC3T3-E1 cells were seeded as previously described and exposed for the period of $48 \mathrm{~h}$. The scaffolds were rinsed twice with PBS and fixed in 3\% glutaraldehyde for $3 \mathrm{~h}$, then rinsed in $0.1 \mathrm{~mL}$ of phosphate-buffered saline. Thereafter, the scaffolds were dehydrated with upgrading concentrations of ethanol $(30 \%, 50 \%, 60 \%, 70 \%, 80 \%, 90 \%, 95 \%$ and $100 \%)$ twice (15 min each). After final washing with $100 \%$ ethanol, all of the samples were kept in fume hood. Finally, the scaffolds were sputtering-coated with gold and observed under SEM to examine the morphology of MC3T3-E1 cells.

Electrospun neat PLA and PLA/HA/GO fibrous scaffolds were used for cytocompatibility tests. The samples were placed into a 24-well polystyrene cell culture plate and soaked with $2 \mathrm{~mL} /$ cell suspension in a $5 \% \mathrm{CO}_{2}$ incubator at $37^{\circ} \mathrm{C}$ for 24 and $48 \mathrm{~h}$, respectively. Before cells seeding, the samples were sterilized by $75 \%$ ethanol, followed by air-drying at room temperature. Finally the cells were stained with Acridine Orange (AO), which was cleaved to yield a green fluorescent product by metabolically active cells. The density of the cells which adhered on each scaffold was measured from randomly selected views of each film observed at 100-fold magnification with a fluorescent microscopy (Olympus BX51).

\section{$1.8 \quad$ MTT assay}

Cell growth on both nanomembrane types was measured by MTT assay. The cell-membrane constructs were cultured for 24 and $48 \mathrm{~h}$. Briefly, $1 \mathrm{~mL}$ MTT $(5 \mathrm{mg} / \mathrm{mL})$ stock solution was added to each well and incubated for $4 \mathrm{~h}$ at $37^{\circ} \mathrm{C}$. Then, remove the medium carefully, and at end of the assay, the blueformazan reaction product was dissolved by adding $750 \mu \mathrm{L}$ DMSO each well and transferred to a 96-well plate. The absorbance was measured by using a microplate reader at wavelength $490 \mathrm{~nm}$, blanked with dimethyl sulfoxide solution. Three replicates were read for each sample; the mean value was used as the final result.

\section{Results and discussion}

\subsection{Characterization of GO}

As shown in Figure 1(a), as-prepared GO sheets were nearly transparent under electron irradiation, indicating the GO sheets were quite thin. The C1s XPS spectrum of GO (Figure 1(b)) indicates the presence of four components: the $\mathrm{C}$
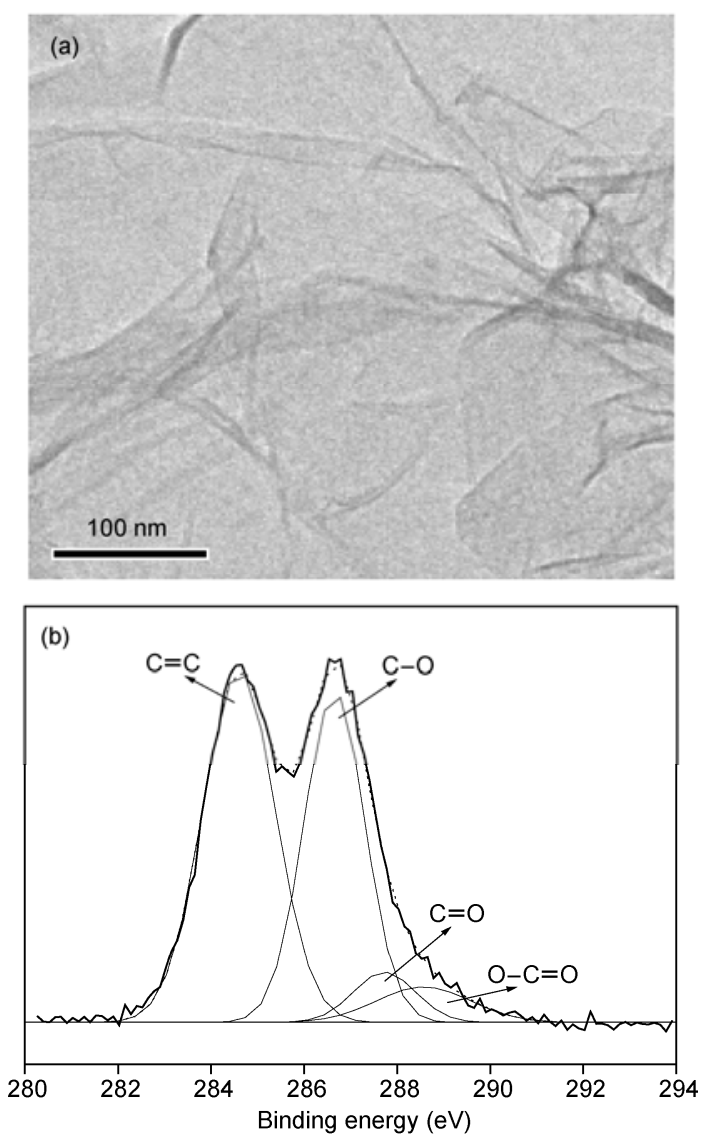

Figure 1 Characterization of GO: (a) TEM image and (b) C1s XPS spectrum. 
in $\mathrm{C}=\mathrm{C}$ bonds $(284.5 \mathrm{eV})$, the $\mathrm{C}$ in $\mathrm{C}-\mathrm{O}$ bonds $(286.6 \mathrm{eV})$, the $\mathrm{C}$ in $\mathrm{C}=\mathrm{O}$ bonds $(287.7 \mathrm{eV})$ and the $\mathrm{C}$ in $\mathrm{O}-\mathrm{C}=\mathrm{O}$ bonds $(288.7 \mathrm{eV})$. It indicates the considerable degree of the oxidation existing in GO material and the hydrophilicity of GO are owning to the existence of the oxygen functionalized groups.

\subsection{Morphology of fibrous scaffolds}

Figure 2 shows the SEM images of electrospun PLA and PLA/HA/GO nanofibers. Ultra-fine fibers were successfully fabricated via electrospinning and all fibers presented loose three-dimensional (3D) frameworks. The difference among two nanofibers was in the following aspects: neat PLA nanofibers exhibited uniform diameter with relatively smooth surface (Figure 2(b) and (d); Adding of HA-GO (Figure 2(a) and (c)) into PLA resulted in the increase of the surface roughness of obtained composite fibers. Especially for PLA/ HA/GO composite nanofibers, there were some joints and protuberances existing in fibers (Figure 2(a)), which were related to the introduction of GO and HA nanoparticles. The integration of 3D framework composed of PLA/HA/GO nanofibers would create a potential scaffold for promoting cell adhesion and growth [42].

\subsection{XRD analyses}

Figure 3 shows the XRD patterns of fibrous PLA, PLA/HA and PLA/HA/GO membranes as well as the XRD patterns of powdery HA and GO. Overall, the shapes of the XRD patterns obtained from the composite nanofibers were similar to that of neat PLA nanofibers, apart from the weakening of PLA diffraction peaks in the composite nanofibers. After adding inorganic compounds (HA or GO), PLA molecules wrapped these inorganic particles, resulting in that the crystallization of PLA phase in the composite fibers was destroyed to a certain degree. With the increase of the amount of inorganic phase, this destruction degree was more obvious. Therefore, the weakening of the PLA diffraction peaks in the PLA/HA/GO nanofibers was the largest. Moreover, as shown in the XRD pattern of the PLA/HA/GO nanofibers in Figure 3, the characteristic peaks attributed to HA phase was very weak and the characteristic peaks attributed to GO phase disappeared. The weakening of HA peaks and the absence of GO peaks were due to two aspects: one was the relatively low contents of $\mathrm{HA}$ and GO, another was the good dispersion of HA and GO within PLA matrix.

\subsection{FTIR analyses}

Figure 4 shows the FTIR spectra of powdery HA and GO, and as-electrospun PLA, PLA/HA and PLA/HA/GO nanofibers. For the GO sample, the characteristic vibrations included the broad and intense $\mathrm{O}-\mathrm{H}$ peak at $3400 \mathrm{~cm}^{-1}$, strong $\mathrm{C}=\mathrm{O}$ peak in carboxylic acid and carbonyl moieties at 1732 $\mathrm{cm}^{-1}, \mathrm{C}-\mathrm{OH}$ peak at $1365 \mathrm{~cm}^{-1}, \mathrm{C}-\mathrm{O}-\mathrm{C}$ peak at $1246 \mathrm{~cm}^{-1}$,
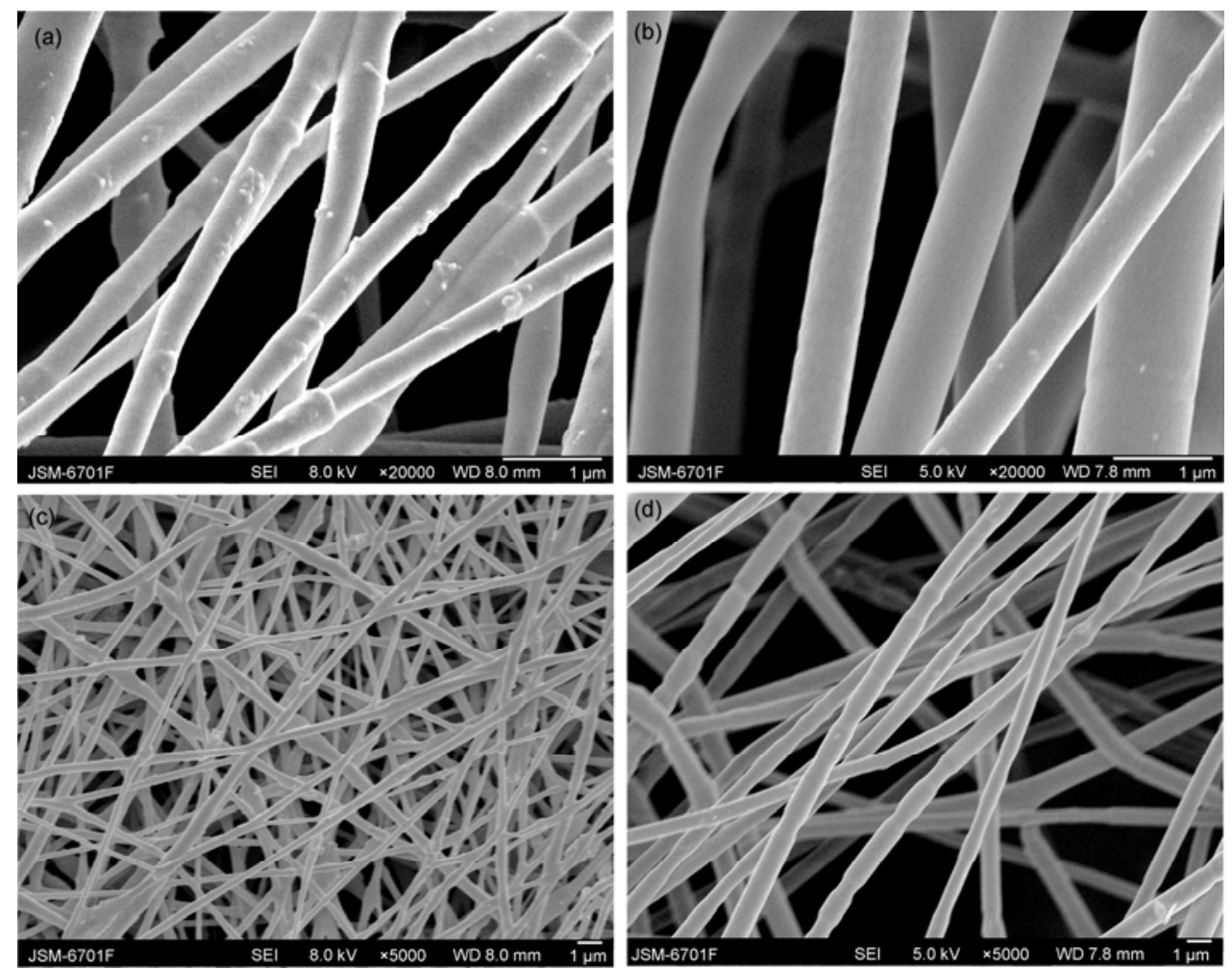

Figure 2 SEM images of PLA/HA/GO nanofibers with high magnification (a) and low magnification (c), electrospun PLA with high magnification (b) and low magnification $(\mathrm{d})$. 


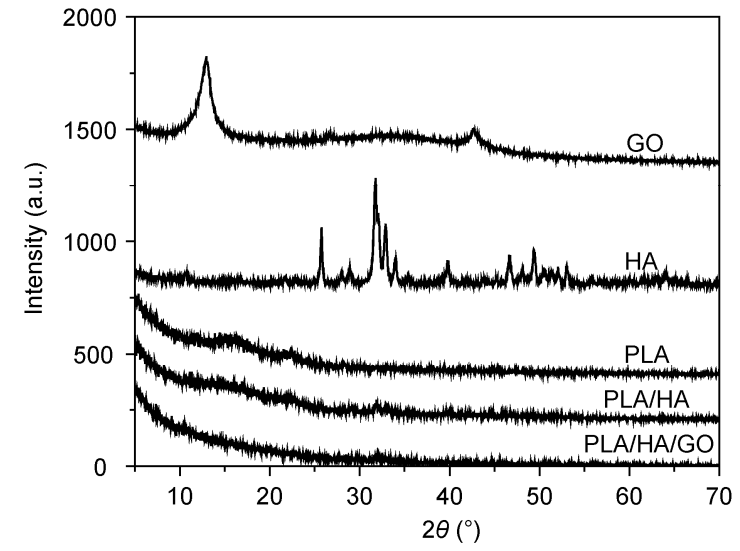

Figure 3 XRD patterns of powdery GO and HA, and fibrous PLA, PLA/ HA and PLA/HA/GO.

C-O stretching peak at $1065 \mathrm{~cm}^{-1}$ and aromatic $\mathrm{C}-\mathrm{H}$ peak at $832 \mathrm{~cm}^{-1}$. The peak centered at $1620 \mathrm{~cm}^{-1}$ was assigned to adsorbed water molecules, but might be also containing components from skeletal vibrations of unoxidized graphitic domains [43]. As shown in Figure 4, the main spectrum characteristic of PLA/HA/GO nanofibers was very similar to that of PLA nanofibers, which was due to overlap of the vibration bands of HA and GO ranging from 1800 to 1000 $\mathrm{cm}^{-1}$ with those of PLA. Of course, slight differences between the above two spectra still can be distinguished. Detailed, the intensities of the absorption bands at 1054 and $1620 \mathrm{~cm}^{-1}$ of PLA/HA/GO nanofibers were both stronger than those of PLA nanofibers, which can be attributed to the overlapped effects of HA and GO respectively.

\subsection{Raman analyses}

Raman spectra were employed to investigate the microstructure of powdery PLA, powdery GO, PLA/HA nanofibers and PLA/HA/GO nanocomposite membranes. As shown in Figure 5, GO sample has two prominent peaks at

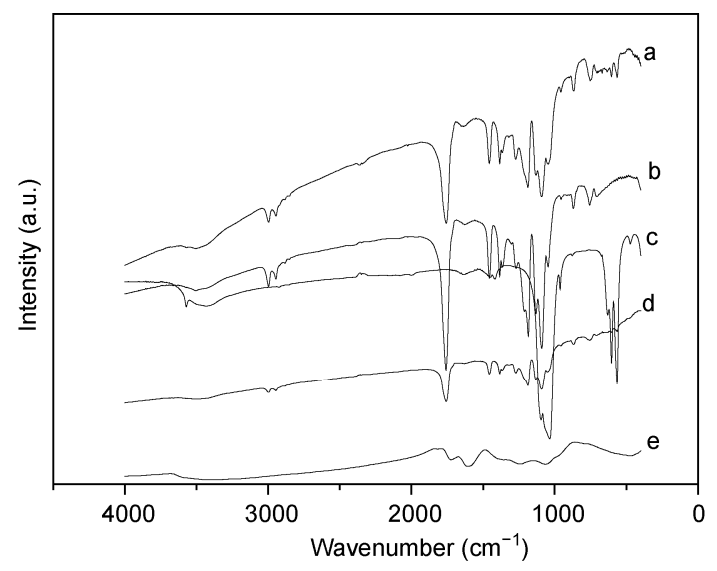

Figure 4 FT-IRRS spectra of PLA, HA, PLA/HA, PLA/HA/GO electropsun nanofibers. (a) PLA/HA/GO nanofibers; (b) PLA nanofibers; (c) HA powders; (d) PLA/HA nanofibers; (e) GO powders.
1580 and $1605 \mathrm{~cm}^{-1}$ corresponding to the $\mathrm{D}$ and $\mathrm{G}$ peaks. It is clear seen that the characteristic D and G peaks of GO present in the Raman spectrum for the PLA/HA/GO sample. It indicates that the GO has been successfully added into PLA/HA/GO nanocomposite membrane.

\subsection{TGA analyses}

Figure 6 presents TGA traces for different fibrous membranes: PLA, PLA/HA and PLA/HA/GO. It is obvious that the curves of all nanofibers kept stable without significant weight loss up to $250^{\circ} \mathrm{C}$, and the residual rates (at $800^{\circ} \mathrm{C}$ ) were roughly estimated to be about $0,13.4 \%$, and $18.7 \%$, respectively. Also, one major stage of weight loss for the three samples, between 250 and $400^{\circ} \mathrm{C}$, was identified and the maximum decomposition temperature was 228.9, 336 and $338.8^{\circ} \mathrm{C}$, respectively. Therefore, the introduction of $\mathrm{HA}$ and GO phases increased the thermal stability of neat PLA nanofibers. We believe that, during the mixing and electrospinning processes, HA nanoparticles and GO nanosheets would interact with PLA molecules by hydrogen bonds and/or with van der Waals forces. The interface actions between organic PLA phase and inorganic HA and

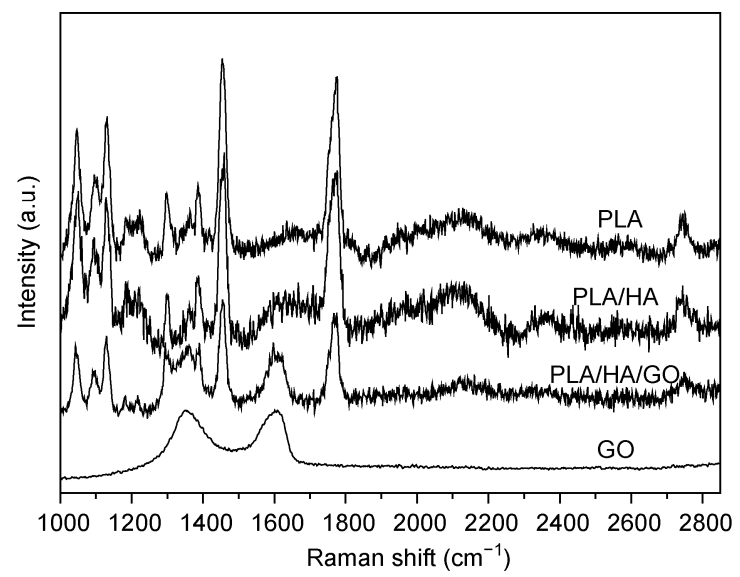

Figure 5 Raman spectra of powdery GO, PLA fibrous, PLA/HA nanofibers and PLA/HA/GO nanocomposite membranes.

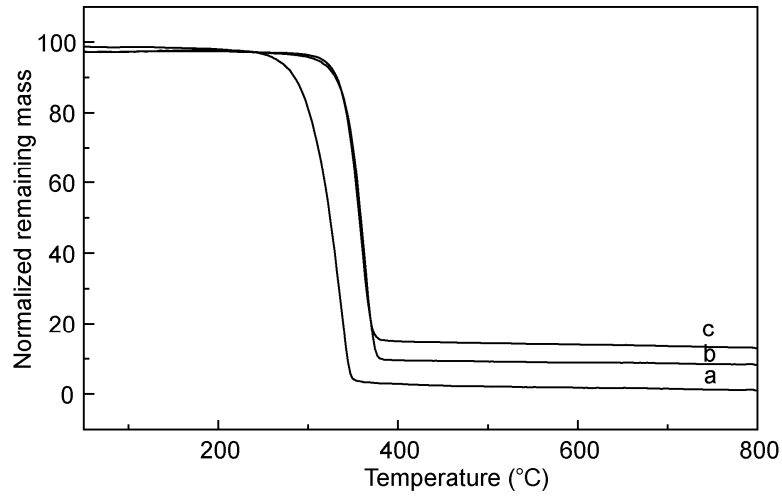

Figure 6 TGA curves of PLA (a), PLA/HA (b) and PLA/HA/GO (c) nanofibers. 
GO phases would be beneficial to the enhancement of the thermal stability of PLA/HA/GO nanofibers.

\subsection{Cytocompatibility tests}

To study the cytocompatibility of the fibrous samples, MC3T3-E1 cells were used in our system, which is commonly used to assess cytotoxicity of potential substrates for cell growth. As shown in Figure 7, after the cells were seeded directly on fibrous scaffolds and incubated for $48 \mathrm{~h}$, the surfaces of three fibrous scaffolds appeared to be covered with cells as well as the ECM secreted by cells. These cells were not only able to spread and extend on scaffold surfaces, but also able to penetrate inside the meshes of the scaffolds. In addition, the cells grown on PLA/HA and PLA/HA/GO scaffolds tended to be much more mature compared with those grown on neat PLA scaffold. Moreover, as easily might find from Figure 7(b), when GO added into the PLA/HA matrix, the cells indicates a plumper appearance compared with those grown on neat PLA scaffold.

Also, this is clearly appeared some pseudopods existed in the PLA/HA/GO nanocomposite fibers, which are probablely produced from cells (arrows in Figure 7(b)). Due to their flat shapes, graphene oxide nanostructures were expected to have even stronger interaction with the cellular membranes. Also, surface wettability is affected not only by surface chemistry but also by topographical parameters such as roughness and texture. Therefore, we come to a conclusion

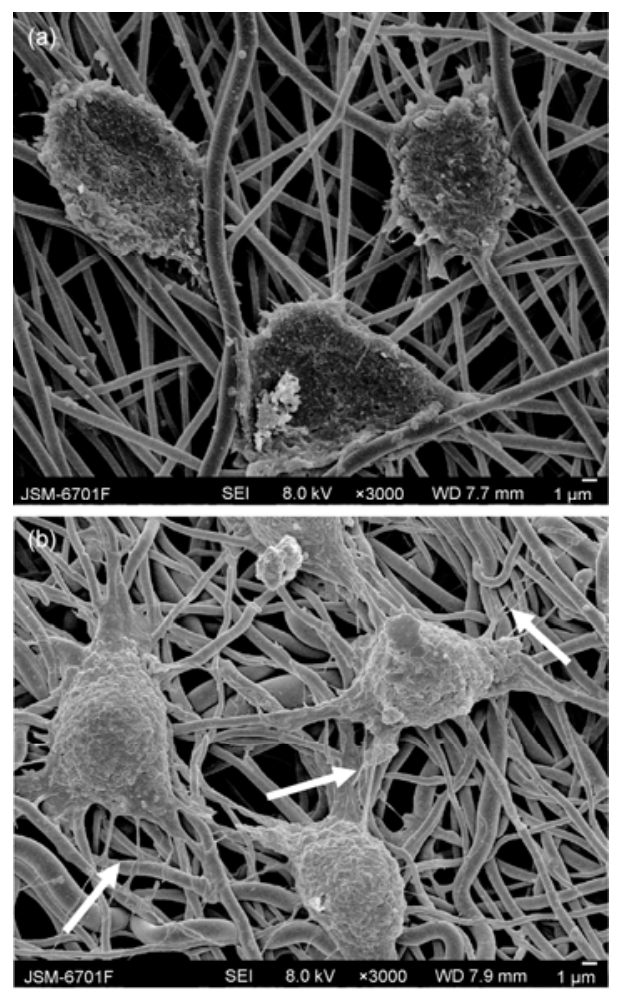

Figure 7 SEM images of MC3T3 E1 cells grown on electrospun PLA nanofibers (a), PLA/HA/GO nanofibers (b). that the addition of GO do not influence the cell viability, and the preservation of biocompatibility for the PLA/ $\mathrm{HA} / \mathrm{GO}$ nanocomposite fibers is attributed to the excellent intrinsic biocompatibility and the hydrophilic nature of GO [33].

The cell growth onto neat PLA and PLA/HA/GO membranes were also determined using fluorescence microscopy. Figure 8(a) and (c) shows the cell attachment on neat PLA scaffold and Figure 8(b) and (d) shows the cell attachment on PLA/HA/GO composite scaffold, in the periods of 24 and $48 \mathrm{~h}$ respectively. It is clear that MC3T3-E1 cells well adhered and proliferated on the two scaffolds with the increase of the culture time. Also, compared with neat PLA scaffold, the amount of the cells grown on PLA/HA/GO scaffold was larger after $48 \mathrm{~h}$ of proliferation.

The osteoblast growth on the nanomembranes at 24 and $48 \mathrm{~h}$ after seeding was determined using the MTT assay (Figure 9). The PLA/HA/GO nanocomposites exhibited significantly higher cell growth compared to PLA scaffolds and PLA/HA scaffolds at $24 \mathrm{~h}$. Osteoblasts showed a slightly higher growth rate on a PLA/HA/GO (5\% GO) scaffolds than on PLA and PLA/HA membranes at $48 \mathrm{~h}$.

Scaffold properties play an active regulating role in controlling the cell attachment and morphology [44], and impose a direct influence on intracellular responses. Cell behavior such as adhesion and proliferation represents the initial phase of cell-scaffold communication that subsequently effects cell differentiation $[45,46]$. Webster's group $[44,45]$ suggested that nanostructure with high surface area and nanophase calcium phosphate are capable of enhancing osteoblastic proliferation and differentiation. In our system, the porous 3D frameworks composed of electrospun nanofibers could offer the attachment, migration and proliferation of cells towards special directions. HA was able to attract the MC3T3-E1 cells by enhancing the cell capture efficiency [46,47]. GO was also able to support a positive tendency to cell proliferation based on its excellent biocompatibility combined with natural hydrophility [35]. Therefore, based on the above investigations, the PLA/HA/ GO composite nanofibers can offer an ideal biocompatible scaffold for cell culture.

\section{Conclusions}

In summary, we demonstrated that ternary PLA/HA/GO nanofibers could be prepared by simple electrospinning method. The powdery HA and GO were uniformly dispersed into PLA matrix and the obtained nanocomposite material maintained the fibrous 3D framework. Furthermore, the PLA/HA/GO nanofibers displayed better biocompatibility compared with neat PLA nanofibers. We believe that, the combined advantages of PLA/HA/GO scaffold, including porous fibrous 3D structure, biocompatibility and mechanical properties coming from HA and GO, will makes such a 

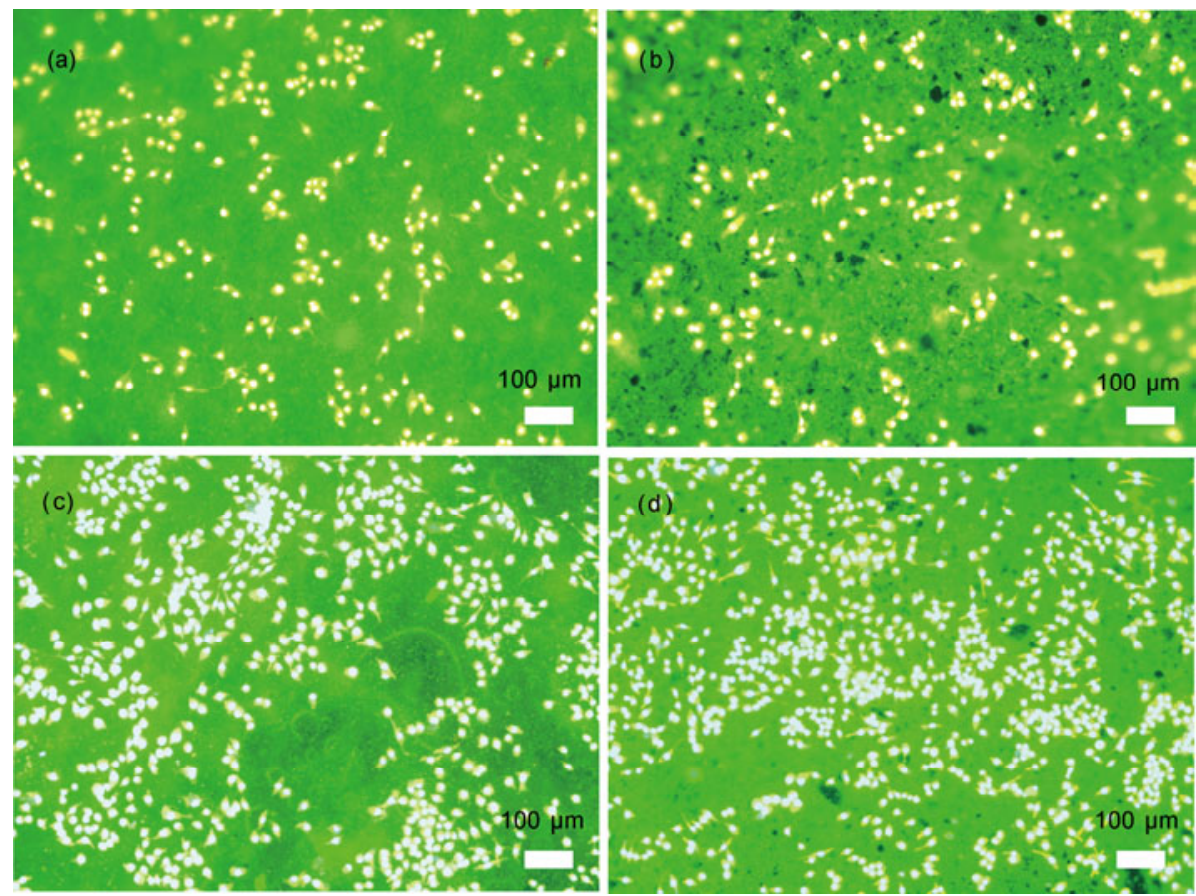

Figure 8 (Color online) Fluorescent microscopy images of MC3T3-E1 cells grown on PLA nanofibers for $24 \mathrm{~h}$ (a) and $48 \mathrm{~h}$ (c), and grown on PLA/HA/ GO nanofibers for $24 \mathrm{~h}(\mathrm{~b})$ and $48 \mathrm{~h}$ (d) respectively.

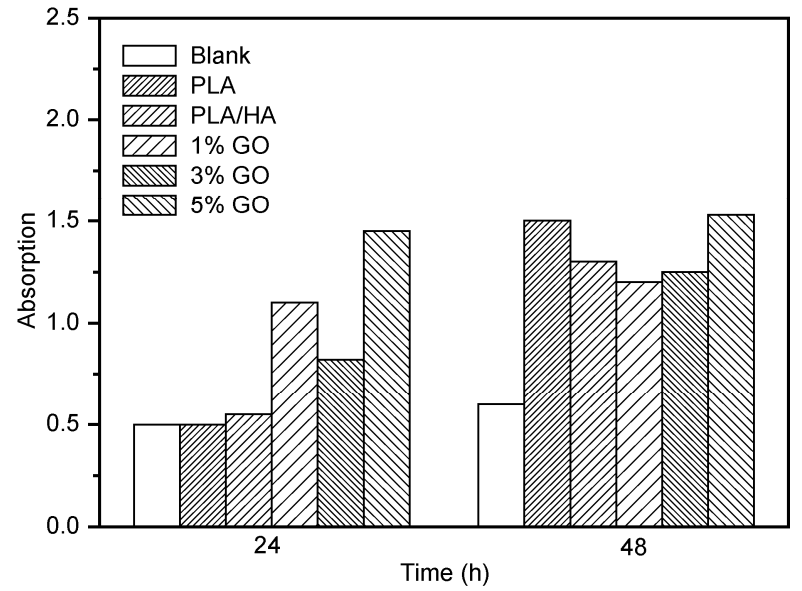

Figure 9 MC3T3-E1 cell proliferation on nanofiber membranes for 24 and $48 \mathrm{~h}$.

kind of nanocomposite fibrous scaffold as a promising material for tissue engineering applications.

This work was supported by the Hundred Talents Program of Chinese Academy of Sciences and the National Natural Science Foundation of China (51005225).

1 Langer R, Vacanti J. Tissue engineering. Science, 1993, 260: 920-926

2 Crane G M, Ishaug S L, Mikos A G. Bone tissue engineering. Nat Med, 1995, 1: 1322-1324

3 Yaszemski M J, Payne R G, Hayes W C, et al. Evolution of bone transplantation: Molecular, cellular and tissue strategies to engineer human bone. Biomaterials, 1996,17: 175-185

4 Vacanti C A, Vacanti J P. Bone and cartilage reconstruction with tissue engineering approaches. Otolaryngol Clin North Am, 1994, 27: 263-276

5 Devin J E, Attawia M A, Laurencin C T. Three-dimensional degradable porous polymer-ceramic matrices for use in bone repair. $\mathrm{J}$ Biomater Sci Polymer Edn, 1996, 7: 661-669

6 Marra K G, Szem J W, Kumta P N, et al. In vitro analysis of biodegradable polymer blend/ hydroxyapatite composites for bone tissue engineering. J Biomed Mater Res, 1999, 47: 24-35

7 Maquet V, Boccaccini A R, Pravata L, et al. Porous poly (a-hydroxyacid)/bioglass composite scaffolds for bone tissue engineering. I: Preparation and in vitro Characterisation. Biomaterials, 2004, 25: 4185-4194

8 Yang F, Murugan R, Ramakrishna S, et al. Fabrication of nanostructured porous PLLA scaffold intended for nerve tissue engineering. Biomaterials, 2004, 25: 1891-1900

9 Russias J, Saiz E, Nalla R K, et al. Fabrication and mechanical properties of PLA/ HA composites: A study of in vitro degradation. Mater Sci Engineer C, 2006, 26: 1289-1295

10 Lannuttia J, Reneker D, Ma T, et al. Electrospinning for tissue engineering scaffolds. Mater Sci Engineer C, 2007, 27: 504-509

11 Liu C, Xia Z, Czernuszka J T. Design and development of threedimensional scaffolds for tissue engineering. Trans I ChemE Part A, 2007, 85: 1051-1064

12 Subbiah T, Bhat G S, Tock R W, et al. Electrospinning of nanofibers. J Appl Polym Sci, 2005, 96: 557-569

13 Reneker D H, Chun I. Nanometre diameter fibres of polymer, produced by electrospinning. Nanotechnology, 1996, 7: 216-223

14 Yang Y, Jia Z, Li Q, et al. Electrospinning and its application. High Voltage Engine, 2006, 32: 91-95

15 Qin X H, Wang S Y. Electrospun nanofibers from crosslinked poly (vinyl alcohol) and its filtration efficiency. J Appl Polym Sci, 2008, 109: 951-956

16 Mo X M, Xu C Y, Kotaki M, et al. Electrospun P (LLA-CL) nanofiber: A biomimetic extracellular matrix for smooth muscle cell and endothelial cell proliferation. Biomaterials, 2004, 25: 1883-1890

17 Xu C Y, Inai R, Kotaki M, et al. Aligned biodegradable nanofibrous 
structure: A potential scaffold for blood vessel engineering. Biomaterials, 2004, 25: 877-886

18 Hutmacher D W. Scaffolds in tissue engineering bone and cartilage. Biomaterials, 2000, 21: 2529-2543

19 Li W J, Laurencin C T, Caterson E J, et al. Electrospun nanofibrous structure: A novel scaffold for tissue engineering. J Biomed Mater Res, 2002, 60: 613-621

20 Kim H W, Lee H H, Knowles J C. Electrospinning biomedical nanocomposite fibers of hydroxyapaite/poly (lactic acid) for bone regeneration. J Biomed Mater Res Part A, 2006, 79A: 643-649

21 Montjovent M O, Mathieu L, Hinz B, et al. Biocompatibility of bioresorbable poly (L-lactic acid) composite scaffolds obtained by supercritical gas foaming with human fetal bone cells. Tissue Eng, 2005, 11: 1640-1649

22 Schmack G, Tandler B, Vogel R, et al. Biodegradable fibers of poly (L-lactide) produced by high-speed melt spinning and spin drawing. $\mathrm{J}$ Appl Polym Sci, 1999, 73: 2785-3000

23 Fujuan L, Rui G, Mingwu S, et al. Effect of the porous microstructures of poly(lactic-co-glycolic acid)/carbon nanotube composites on the growth of fibroblast cells. Soft Mater, 2010, 8: 239-253

24 Furuzono T, Kishida A, Tanaka J. Nano-scaled hydroxyapatite/polymer composite I coating of sintered hydroxyapatite particles on poly $(\gamma$-methacryloxypropyl trimethoxysilane)-grafted silk fibroin fibers through chemical bonding. J Mate Sci Mater Med, 2004, 15: 19-23

25 Wang S, Tambraparni M, Qiu J J, et al. Thermal expansion of graphene composites. Macromolecules, 2009, 45: 5251-5255

26 Jiang J W, Wang J S, Li B. Young's modulus of graphene: A molecular dynamics study. Phys Rev B, 2009, 80: 113405-113408

27 Shi Y M, Fang W J, Zhang K K, et al. Photoelectrical response in single-layer graphene transistors. Small, 2009, 5: 2005-2011

28 Cai D Y, Song M. A simple route to enhance the interface between graphite oxide nanoplatelets and a semi-crystalline polymer for stress transfer. Nanotechnology, 2009, 20: 315708

$29 \mathrm{Xu} \mathrm{Z}$, Gao C. In situ polymerization approach to graphene-reinforced nylon-6 composites. Macromolecules, 2010, 43: 6716-6723

$30 \mathrm{Xu}$ Y, Hong W J, Bai H, et al. Strong and ductile poly (vinyl alcohol)/ graphene oxide composite films with a layered structure. Carbon, 2009, 47: 3538-3543

31 Veca L M, Lu F S, Meziani M J, et al. Polymer functionalization and solubilization of carbon nanosheets. Chem Commun, 2009, 14: 2565-2567

32 Salavagione H J, Gomez M A, Martinez G. Polymeric modification of graphene through esterification of graphite oxide and poly(vinyl alcohol). Macromolecules, 2009, 42: 6331-6334

33 Yan X B, Chen J T, Yang J, et al. Fabrication of free-standing, electrochemically active, and biocompatible graphene oxide-polyaniline and graphene-polyaniline hybrid papers. ACS Appl Mater Interfaces, 2010, 2: 2521-2529

$34 \mathrm{Xu} \mathrm{Z,} \mathrm{Gao} \mathrm{C.} \mathrm{Graphene} \mathrm{chiral} \mathrm{liquid} \mathrm{crystals} \mathrm{and} \mathrm{macroscopic} \mathrm{as-}$ sembled fibres. Nat Commun, 2011, 2: 571

$35 \mathrm{Xu} \mathrm{Y} \mathrm{X,} \mathrm{Bai} \mathrm{H,} \mathrm{Lu} \mathrm{G} \mathrm{W,} \mathrm{et} \mathrm{al.} \mathrm{Flexible} \mathrm{graphene} \mathrm{films} \mathrm{via} \mathrm{the} \mathrm{filtra-}$ tion of water-soluble noncovalent functionalized graphene sheets. J Am Chem Soc, 2008, 130: 5856-5857

$36 \mathrm{Xu} \mathrm{J} \mathrm{Y,} \mathrm{Hu} \mathrm{Y,} \mathrm{Song} \mathrm{L,} \mathrm{et} \mathrm{al.} \mathrm{Preparation} \mathrm{and} \mathrm{characterization} \mathrm{of} \mathrm{poly}$ (vinyl alcohol)/graphite oxide nanocomposite. Carbon, 2002, 40: 445-467

37 Chen Y F, Qi Y Y, Tai Z X, et al. Preparation, mechanical properties and biocompatibility of graphene oxide/ultrahigh molecular weight polyethylene composites. J Eur Polym, 2012, 48: 1026-1033

38 Xiao Y Z, Ji L Y, Cheng P, et al. Distribution and biocompatibility studies of graphene oxide in mice after intravenous administration. Carbon, 2011, 49: 986-995

39 Kan W, Jing R, Hua S, et al. In vitro toxicity evaluation of graphene oxide on A549 cells. Tox Lett, 2011, 200: 201-210

40 Wang K, Ruan J, Song H, et al. Biocompatibility of graphene oxide. Nanoscale Res Lett, 2011, 6: 1-8

41 William S, Hummers J, Richard E O. Preparation of graphitic oxide. J Am Chem Soc, 1958, 80: 1339

42 Stankovich S, Piner R D, Nguyen S T, et al. Synthesis and exfoliation of isocyanate-treated graphene oxide nanoplatelets. Carbon, 2006, 44: 3342-3347

43 Kim B, Mooney D J. Development of biocompatible synthetic extracellular matrices for tissue engineering. Trend Biotech, 1998, 16: 224-230

44 He W, Yong T, Teo W E, et al. Fabrication and enthothelialization of collagen-blended biodegradable polymer nanofibers: Potential vascular graft for the blood vessel tissue engineering. Tissue Eng, 2005, 11: $1574-1588$

45 Webster T J, Ergun C, Doremus R H, et al. Enhanced functions of osteoblasts on nanophase ceramics. Biomaterials, 2000, 21: 18031810

46 Hutmacher D W. Scaffolds in tissue engineering bone and cartilage. Biomaterials, 2000, 21: 2529-2543

47 Cui Y, Liu Y, Jing X B, et al. The nanocomposite scaffold of poly (lactide-co-glycolide) and hydroxyapatite surface-grafted with L-lactic acid oligomer for bone repair. Acta Biomaterialia, 2009, 5: 2680-2692

Open Access This article is distributed under the terms of the Creative Commons Attribution License which permits any use, distribution, and reproduction in any medium, provided the original author(s) and source are credited. 